\title{
ANTIBACTERIAL ACTIVITIES OF CULTURAL FILTRATES OF SOME STRAINS OF MICROMYCETE
}

\author{
I.G. RUBEZHNIAK \\ National University of Life and Environmental sciences of Ukraine \\ Kyiv Ukraine \\ ORCID ID: https://orcid.org/0000-0003-1875-8963
}

\begin{abstract}
Annotation. The purpose of this work was conducting study of the antibacterial activity of cultural filtrates of 125 micromycete strains isolated from different samples. Different Gram-negative and Gram-positive bacteria as test-organisms have been applicable. Activity of 64 strains has been established. Genus Aspergillus and Penicillium was characterized wide spectrum of activity. The strains of genus Aureobasidium and Paecilomyces as $A$. pullulans $41, P$. variotii 68 have been shown antibacterial action against test-organisms. It is found that strains of $M$. vinacea exhibited antibacterial activity against Staphylococcus aureus 904 and E. coli 906. Most strains did not show antibacterial effect. The results obtained confirm that antibacterial potential of strains of A. pullulans, P. variotii and $M$. vinacea is the most potential for researches of biologically active substances.
\end{abstract}

Keywords: micromycetes, antibacterial activity, Gram-negative bacteria, Grampositive bacteria

\section{Introduction.}

The micromycetes have been famous as producers of antibiot.es and other secondary biologically active metabolites as vitamins, attractants, cytotoxins, insecticides, compounds that promote or inhibit growth, etc. [1, 2]. The strains of micromycetes have been traditionally used to produce a variety of important substances for the pharmaceutical and food industries.

System researches concerning ability to produce the biologically active substances were conducted by different authors on the separate strains of molds $[2,3]$. Wide potential of micromycetes had not been ful- ly studied up to now. Screening of novel strains arc bringing about microorganisms, not yet full assayed for their antibacterial activity that can produce useful templates with new antibiotics activity.

The purpose of our work was study of antibacterial activity of cultural filtrates of 125 micromycete strains as a starting point for father investigations of the most perspective strains for feasible obtaining of antibacterial substances.

\section{Materials and methods.}

In this work it was used 125 micromycete strains of the genus Aspergillus, Alter- 
naria, Aureobasidium, Beauveria, Botrytis, Chaetomium, Cladosporium, Curvularia, Endomyces, Fusarium, Gliocladium, Mortierella, Nigrospora, Paecilomyces, Penicillium, Pseudallescheria, Scopulariopsis, Trichoderma, Ulocladium and separate strains Nectria sp., Phialophora sp. and Verticillium dahlia, which were isolated from different ecological niches such as air, dwelling walls, soil of Chernigovska area and rhizosphere of plants.

The micromycetes were grown on a basic nutritive Czapek medium [5]. The culture liquid filtrates were tested against Gram-positive bacteria as Staphylococcus aureus 904, Bacillus licheniformis 5 and Gram-negative bacteria as Escherichia coli 906, Agrobacterium tumefaciens 8464, Pectobacterium carotovorum 8636. A. tumefaciens causes crown gall disease on various plant species and $P$. carotovorum is a ubiquitous plant pathogen with a wide host range (carrot, potato, tomato, leafy greens, etc.). B. licheniformis 5 is a bacterium that is commonly found in soil and bird feathers. These bacteria are known to cause food poisoning and food spoilage. E. coli lives in the intestines of humans and helps keep our guts healthy. But certain strains of $E$. coli can cause severe illness such as urinary tract infections and neonatal sepsis, represent a huge public health problem. Bacteria $S$. aureus is a human pathogen that causes a wide variety of different infections. Bacterial test organisms have beat incubated on the medium of agarose gel for 24 hours at $28^{\circ} \mathrm{C}$.

The screening study of culture liquid filtrate of micromycete strains using standard agar well diffusion method was followed. Eight-millimeter diameter wells were cut from the agar using a sterile cork-borer and $100 \mu \mathrm{l}$ of filtrate were delivered into the wells [5]. A di- ameter of inhibition zones of bacteria was measured after18-24 hours.

Bacterial strains were collected from the Department of Physiology and Taxonomy of Micromycetes, Danylo Zabolotny Institute Microbiology and Virology NAS of Ukraine.

\section{Results.}

It can be seen in fable 1 that only 64 from 125 strains shown antibacterial activity and inhibit the growth of test organisms. Among them, strains such as A. pullulons 41, P. variotii 68 . P. brevicompactum 144 have a broad spectrum of antibiotic activity against both phytopathogenic bacteria and nonphytopathogenic bacteria. The separate strains such as A. ustus 103, A. terreus $119, T$. viride 120 and the strains of the genus Penicillium as P. aculeatum 121.P. ochrochloron 145 and P. purpurogenum 143 showed activity against four from live examined bacteria. The majority strains exhibited antibacterial activity against three different bacteria.

The study of antibiotic effect of micromycete strains against $B$. licheniformis 5 indicated that $A$. alleaceus 118 , A. versicolor 56, A pullulans 4346, C. cladosporiodes 8, G. virens 39, C. inaequalis 84, F. merismoides 151, M. vinacea $62,81-82$, P. variotii $67,69, T$. harzianum 157 and separate strains of the genus Penicillium as P. funiculosum 2, $P$. chrysogenum 163 and P. rubrum 91 didn't visualize antibacterial effect. Other strains inhibited growth of $B$. licheniformis 5 (inhibition zone from 24 to $8,7 \mathrm{~mm}$ ).

The result of study indicated that strains as $A$. versicolor $56, F$. solani 128, $M$. vinacea 81-82 inhibited the growth of human pathogen strain $E$. coli 906 only (zone of inhibition from 25 to $11 \mathrm{~mm})$. 
The separate strains such as $A$. alleaceus 118, A. pullulans 43-46, P. rubrum 91 displayed antibiotic activity against $P$. carotovorum only. These strains didn't show antibiotic affect against tested E. coli, S. aureus and B. licheniformis. The strain $G$. virens 39 shown antibiotic activity against $A$. tumefaciens (inhibition zone is $18 \mathrm{~mm}$ ).

The strains of B. bassiana, C. globosum, F. culmorum, Fusarium sp., Nectria sp., N. oryzae, P. ucrainicum, S. brumptii, T. brevicompacium, Trichoderma sp. and U. atrum did not display antibiotic activity.

\section{Discussion.}

The finding shows that micromycete strains produce many bioactive compounds as secondary metabolites including antibiotics and toxins. The results obtained demonstrated that representatives of the genus Aspergillus and genus Penicillium arc characterized by high antibiotic activity against phytopathogen bacteria and $B$. licheniformis 5. Only some strains of genus Aspergillus as A. parvulus 30, A. terreus 119, A versicolor $56, A$. ustus 103 and genus Penicillium as $P$. aculeatum 123, P. brevicompactum 144, $P$. purpurogenum 143 and $P$. ochrochloron 145 have antibiotic activity against $S$. aureus 904 and E. coli 906 or one of them.

Our experiments are consistent with previous results [6, 7]. Tsyganenko shown that Aspergillus strains demonstrated antibiotic activity especially A. parvulus 3142 [6, 7]. Some authors have also suggested that most of the Aspergillus strains displayed antimicrobial activity against methicillinresistant $S$. aureus, extended-spectrum beta-lactamase-producing $E$. coli $[8]$. A large number of fungal extracts and/or extracellular products have been found to have antimicrobial activity, mainly from the filamentous fungus Penicillium sp. $[9,10]$. The Penicillium species shown distinguished antimicrobial activities towards Candida albicans, B. subtillis, S. aureus, Salmonella typhi and E. coli [11]. Metabolites as penicillanthone and penicillidic acids A-C from strain of

$P$. aculeatum PSU-RSPG105 showed moderate antibacterial activity against E. coli and $S$. aureus [12].

The extracts of cultural filtrates of A. niveus 2411 and Penicillium sp. 1051 have been shown the activity concerning phytopathogenic bacteria $A$. tumefaciens 8464 . Note should be taken that the traditional methods of controlling of phytopathogenic bacteria such as metalcontaining pesticides were not effective [13]. The fact of antibacterial activity of fungal culture filtrates against $A$. tumefaciens 8464 and $P$. carotovorum 8636 has been established previously [14]. Of great interest for the researches is the subsequent investigation of antibiotic activity of Aspergillus and Penicillium species as A. alleaceus 118, A. pullulans 4346, $P$. rubrum 91 shown phytopathogenic activity against $P$. carotovorum 8636 and $A$. versicolor 56 against $E$. coli 906 .

Another promising finding was that species of the genus Aureobasidium and Paecilomyces genus are characterized by antibacterial activity. Two strains such as $A$. pullulans 41, P. variotii 68 shown antibacterial effect against all test bacterial strains. It was reported in literature that strains of $A$. pullulans produce a wide range of substances such as antimicrobial chemicals, siderophores, enzymes, polysaccharides, polyesters and heavy oils [15]. In addition, an A. pullulans strain can produce antimicrobial compounds towards the Gram-negative Pseudomonas fluorescens and Gram-positive S. au- 
reus bacteria [16]. The antibacterial activity of $A$. pullulans strains can be associated with 2-propylacrylic acid, 8,9-dihydroxy-2-methyl-4H,5H-pyrano [3,2-c]-chromon-4-one, 2-methylenesuccinic acid and hexane- 1,2,3.5,6- hexol [17]. Equally important, some strains of $A$. pullulans are used in biological control of plant and storage diseases [18, 19]. In recent years there has been considerable interest in strains of $\mathrm{A}$. pullulans as biopesticides. A proprietary mixture of two strains of $A$. pullulans was recently registered in the U.S. under the trade name "Blossom Protect" as a biocontrol to prevent blossom infections by Erwinia amylovora, the fire blight pathogen Blossom Protect has been used successfully both Europe and in the Pacific Northwest. The new Botecior Fungicide contains yeast-like fungi, A. pullulans strains DSM 14940 and DSM 14941, as the active constituent [20].

The authors of more studies of secondary metabolites of $P$. variotii have exhibited that Gram-positive bacteria Enterococcus faecalis were inhibited by fungal extract [21]. Paeciloketals (1-3), new benzannulated spiroketal derivatives, were isolated from the marine fungus $P$. variotii derived from the giant jellyfish Nemopilema nomurai. Compound 1 showed modest antibacterial activity against the marine pathogen Vibrio ichthyoenteri [22].

There have been several reports of antibiotic potential of $M$. vinacea. The cultural filtrates certain strains $M$. vinacea have been shown antibacterial activity against phytopathogenic bacteria A. tumefaciens and P. carotovorum [14]. The known compound from $M$. vinacea methyl 2,4-dthydroxy-3,5,6-trimethylbenzoate, mortivinacins $\mathrm{A}$ and nicotinic acid were responsible for the antibacterial activities of the extract [23].

The results obtained confirm that cultural filtrates of strains of $A$. pullulans, $P$. variotii and $M$. vinacea possess marked antibacterial activity. It must also be noted that it is very little data on antibiotic effect of strains of $A$. pullulans, M. vinacea and P. variotii. The evidence suggests that these strains are potentially important set of targets for farther investigation.

\section{Antimicrobial activity of cultural liquid of micromycetes}

\begin{tabular}{|c|c|c|c|c|c|c|c|}
\hline & \multirow{4}{*}{ Species } & \multirow{4}{*}{ Strains } & \multicolumn{5}{|c|}{ Diameter of inhibition zones of test, $\mathrm{mm}$} \\
\hline & & & \multicolumn{5}{|c|}{ Bacterium } \\
\hline \multirow{2}{*}{ z } & & & \multicolumn{2}{|c|}{ Gram positive } & \multicolumn{3}{|c|}{ Gram negative } \\
\hline & & & 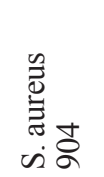 & 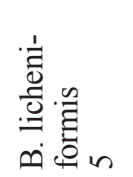 & \begin{tabular}{l}
8 \\
$:$ \\
\hdashline \\
0 \\
0 \\
1
\end{tabular} & 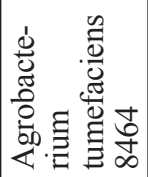 & 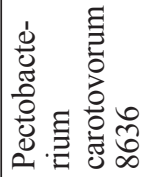 \\
\hline 1 & 2 & 3 & 4 & 5 & 6 & 7 & 8 \\
\hline 1 & $\begin{array}{l}\text { Altemaria } \\
\text { alternata }\end{array}$ & 116 & 0 & 0 & 0 & $16,0 \pm 0,7$ & $11,0 \pm 0,9$ \\
\hline 2 & $\begin{array}{l}\text { Aspergillus } \\
\text { alliaceus }\end{array}$ & 118 & 0 & 0 & 0 & 0 & $15,0 \pm 1,0$ \\
\hline 3 & A. parvulus & 30 & 0 & $14,7 \pm 0,3$ & $10,3 \pm 0,3$ & $30,0 \pm 0,7$ & 0 \\
\hline 4 & & 31 & 0 & $29,0 \pm 1,5$ & 0 & 0 & $24,0 \pm 2,0$ \\
\hline 5 & A. terreus & 119 & $30 \pm 1,5$ & $29,5 \pm 1,0$ & $28,0 \pm 1,5$ & 0 & $15,0 \pm 2,0$ \\
\hline
\end{tabular}




\begin{tabular}{|c|c|c|c|c|c|c|c|}
\hline \multicolumn{8}{|c|}{ 1. Antimicrobial activity of cultural liquid of micromycetes } \\
\hline 1 & 2 & 3 & 4 & 5 & 6 & 7 & 8 \\
\hline 6 & A. versicolor & 56 & 0 & 0 & $25,0 \pm 1,5$ & 0 & 0 \\
\hline 7 & & 58 & 0 & $23,0 \pm 1,0$ & 0 & 0 & 0 \\
\hline 8 & & 59 & 0 & $14,0 \pm 0,6$ & 0 & 0 & 0 \\
\hline 9 & & 60 & 0 & $17,3 \pm 0,4$ & 0 & $13,0 \pm 1,0$ & 0 \\
\hline 10 & & 61 & 0 & $14,3 \pm 0,4$ & 0 & 0 & $19,0 \pm 3,0$ \\
\hline 11 & A. ustus & 101 & 0 & $15,2 \pm 0,4$ & 0 & 0 & $13,0 \pm 1,0$ \\
\hline 12 & & 102 & 0 & $12,0 \pm 1,0$ & 0 & 0 & $15,0 \pm 2,0$ \\
\hline 13 & & 103 & $15,0 \pm 0,7$ & $16,0 \pm 0,7$ & $19,8 \pm 0,4$ & 0 & $15,0 \pm 1,0$ \\
\hline 14 & $\begin{array}{l}\text { Aureobasidium } \\
\text { pullulans }\end{array}$ & 41 & $10,0 \pm 0,7$ & $15,0 \pm 0,7$ & $11,7 \pm 0,9$ & $30,0 \pm 0,8$ & $25,0 \pm 1,0$ \\
\hline 15 & & 43 & 0 & 0 & 0 & 0 & $15,7 \pm 0,4$ \\
\hline 16 & & 44 & 0 & 0 & 0 & 0 & $10,8 \pm 0,4$ \\
\hline 17 & & 45 & 0 & 0 & 0 & 0 & $20,0 \pm 0,7$ \\
\hline 18 & & 46 & 0 & 0 & 0 & 0 & $20,0 \pm 0,7$ \\
\hline 19 & & 150 & 0 & $25 \pm 2,0$ & 0 & 0 & 0 \\
\hline 20 & Botrytis sp. & 15 & $15,0 \pm 0,3$ & $10,0 \pm 0,7$ & 0 & 0 & 0 \\
\hline 21 & Botrytis cinerea & 55 & $12,0 \pm 1,0$ & $11,0 \pm 0,6$ & $33,7 \pm 1,1$ & 0 & 0 \\
\hline 22 & \begin{tabular}{|l|}
$\begin{array}{l}\text { Cladosporium } \\
\text { cladosporiodes }\end{array}$ \\
\end{tabular} & 8 & 0 & 0 & 0 & $23,5 \pm 4,0$ & $14,5 \pm 4,5$ \\
\hline 23 & & 9 & 0 & $11,0 \pm 0,3$ & 0 & $10,7 \pm 0,3$ & $12,0 \pm 2,0$ \\
\hline 24 & & 12 & 0 & $18,7 \pm 0,6$ & 0 & 0 & 0 \\
\hline 25 & $\begin{array}{l}\text { Gliocladium } \\
\text { virens }\end{array}$ & 39 & 0 & 0 & 0 & $18,0 \pm 1,0$ & 0 \\
\hline 26 & $\begin{array}{l}\text { Curvularia } \\
\text { inaequalis }\end{array}$ & 84 & 0 & 0 & $11,7 \pm 0,4$ & 0 & 0 \\
\hline 27 & Fusarium lactis & 130 & 0 & $18,3 \pm 0,3$ & 0 & 0 & 0 \\
\hline 28 & $\begin{array}{l}\begin{array}{l}\text { F. merismoides f. } \\
\text { merismoides }\end{array} \\
\end{array}$ & 151 & 0 & 0 & 0 & $15,0 \pm 0,3$ & $16,0 \pm 1,0$ \\
\hline 29 & F. solani & 128 & 0 & $14,0 \pm 1.0$ & 0 & 0 & 0 \\
\hline 30 & & 129 & 0 & 0 & $19 \pm 0,7$ & 0 & 0 \\
\hline 31 & $\begin{array}{l}\text { Mortierella } \\
\text { vinacea }\end{array}$ & 62 & 0 & 0 & $20,0 \pm 0,7$ & 0 & $22,0 \pm 1,0$ \\
\hline 32 & & 63 & $12,0 \pm 1,0$ & $14 \pm 1,0$ & 0 & 0 & $24,0 \pm 1.0$ \\
\hline 33 & & 81 & 0 & 0 & $11,3 \pm 0,4$ & 0 & 0 \\
\hline 34 & & 82 & 0 & 0 & $11,7 \pm 0,4$ & 0 & 0 \\
\hline 35 & $\begin{array}{l}\text { Paecilomyces } \\
\text { variotii }\end{array}$ & 67 & 0 & 0 & 0 & $12,7 \pm 0,1$ & $10,0 \pm 1,0$ \\
\hline 36 & & 68 & $14,3 \pm 0,6$ & $17,6 \pm 0,4$ & $12,0 \pm 2,0$ & $30,0 \pm 1,0$ & $20,0 \pm 0,7$ \\
\hline 37 & & 69 & $11,0 \pm 0,7$ & 0 & 0 & 0 & $10,7 \pm 0,8$ \\
\hline 38 & P. lilacinus & 127 & 0 & $24,0 \pm 1,5$ & $20,0 \pm 1,0$ & 0 & 0 \\
\hline 39 & P. marquandii & 126 & 0 & $16,7 \pm 0,4$ & $16,3 \pm 0,4$ & 0 & 0 \\
\hline 40 & & 132 & 0 & $17,7 \pm 0,4$ & 0 & 0 & 0 \\
\hline 41 & $\begin{array}{l}\text { Penicillium } \\
\text { aculeatum }\end{array}$ & 121 & 0 & $20,3 \pm 0.4$ & 0 & 0 & 0 \\
\hline 42 & & 123 & $12,0 \pm 0,7$ & $19,0 \pm 1,0$ & 0 & $12,0 \pm 0,7$ & $14,0 \pm 1,0$ \\
\hline
\end{tabular}




\begin{tabular}{|c|l|c|c|c|c|c|c|}
\hline \multicolumn{7}{|c|}{ 1. Antimicrobial activity of cultural liquid of micromycetes } \\
\hline 1 & 2 & 3 & 4 & 5 & 6 & 7 & 8 \\
\hline 43 & & 124 & 0 & $13,5 \pm 0,5$ & 0 & $20,0 \pm 2,0$ & $11,0 \pm 0,7$ \\
\hline 44 & P. brevicompactum & 144 & $12,7 \pm 0,4$ & $14,0 \pm 1,0$ & $10,7 \pm 0,4$ & $13,0 \pm 2,0$ & $17,0 \pm 2,0$ \\
\hline 45 & P. clavigerum & 112 & 0 & $11,0 \pm 0,7$ & 0 & 0 & 0 \\
\hline 46 & P. funiculosum & 1 & 0 & $12,7 \pm 0,4$ & 0 & $15,0 \pm 2,0$ & $12,0 \pm 0,7$ \\
\hline 47 & & 2 & 0 & 0 & 0 & $32,0 \pm 1,0$ & $9,0 \pm 0,3$ \\
\hline 48 & & 3 & 0 & $24,0 \pm 1,0$ & 0 & $18,0 \pm 0,7$ & $19,3 \pm 0,7$ \\
\hline 49 & & 4 & 0 & $18,3 \pm 0,7$ & 0 & $16,7 \pm 0,4$ & $16,3 \pm 0,4$ \\
\hline 50 & & 5 & 0 & $14,0 \pm 0$ & 0 & $16,3 \pm 0,4$ & $15,3 \pm 0,4$ \\
\hline 51 & & 6 & 0 & $20,3 \pm 0,4$ & 0 & $17,0 \pm 0,7$ & $17,7 \pm 1,1$ \\
\hline 52 & & 7 & 0 & $12,0 \pm 2,0$ & 0 & 0 & $11,0 \pm 0,7$ \\
\hline 53 & P. chrysogenum & 163 & 0 & 0 & 0 & $39,0 \pm 0,7$ & $14,0 \pm 0,7$ \\
\hline 54 & P. ochrochloron & 145 & $11,7 \pm 0,4$ & $19,0 \pm 1,0$ & 0 & $12,0 \pm 0,7$ & $13,7 \pm 0,1$ \\
\hline 55 & & 156 & 0 & $14,3 \pm 0,4$ & & $20,0 \pm 0,7$ & $11,0 \pm 1,3$ \\
\hline 56 & P. purpurogenum & 143 & 0 & $21,0 \pm 0,5$ & $16,7 \pm 0,4$ & $12,0 \pm 1,3$ & $11,7 \pm 0.4$ \\
\hline 57 & P. rubrum & 91 & 0 & 0 & 0 & 0 & $20,0 \pm 1,3$ \\
\hline 58 & Phialophora sp. & 135 & 0 & $22,0 \pm 2,0$ & $13,3 \pm 0,4$ & 0 & 0 \\
\hline 59 & $\begin{array}{l}\text { Pseudallescheria } \\
\text { boydii }\end{array}$ & 131 & 0 & $17,3 \pm 0,6$ & $12,5 \pm 0,5$ & 0 & 0 \\
\hline 60 & T. harzianum & 157 & 0 & 0 & 0 & $23,0 \pm 0,7$ & $17,7 \pm 0,4$ \\
\hline 61 & T. viride & 120 & $12,0 \pm 1,0$ & $15,7 \pm 0,4$ & $11,3 \pm 0,7$ & 0 & $15,0 \pm 0,7$ \\
\hline 62 & $\begin{array}{l}\text { Verticillium } \\
\text { dahliaee }\end{array}$ & 168 & 0 & $14,3 \pm 0,9$ & 0 & $11,0 \pm 0,7$ & $19,0 \pm 0,7$ \\
\hline 63 & U. consortiale & 106 & 0 & $8,7 \pm 0,4$ & 0 & 0 & $15,0 \pm 1,0$ \\
\hline 64 & & 107 & 0 & $14,3 \pm 0,4$ & 0 & 0 & $15,0 \pm 0,7$ \\
\hline
\end{tabular}

\section{References}

1. Mérillon, J-M. \& Ramawat, K.G. (Eds). (2017). Fungal Metabolites. Switzerland: Springer International Publishing.

2. Rubezhniak, I.G. (2011) [Some features of secondary metabolism of microorganisms]. In: Akutina, S.P. (Eds). Selected issues of modern science. Part 3. Monography (pp. 283-310). In: Moscow: Pero. Russian.

3. Tsyhanenko, K.S., Zaichenko, O. M. (2004). Antybiotychni vlastyvosti deiakykh vydiv rodu Aspergillus Mich. [ Antibiotic properties of some species of genus Aspergillus Mich.] Mikrobiol. Z., 66 (4), 56-61.

4. Tsyhanenko, K. S. (2004). Otsinka antybiotychnoho potentsialu Aspergillus parvulus Smith. [ Evolution of biotic potential of Aspergillus parvulus Smith.] Naukovyi visnyk Chernivetskoho un-tu. Ser. Biolohiia., 194, 33-36.

5. Bilay, V.I. (Eds). (1982). [Methods of experimental mycology]. Kiev: Naukova Dumka.

6. Tsyganenko, K.S. The evaluation of antibiotic and toxigenic potential of some species micromycetes of genera Aspergillus Mich. (2005). [author's abstract]. Danilo Zabolotny Institute of Microbiology and Virology of National Academy of Sciences of Ukraine, Kyiv.

7. Tsyhanenko, K.S., Zaichenko, O.M. (2004). Kharakterystyka fitotoksychnykh vlastyvostei Aspergillus parvulus Smith. [Characteristics of phytotoxic properties of Aspergillus parvulus Smith.] Ahroekol. Z., 4, 42-45.

8. Svahn, K. S., Goransson, U., El-Seedi, H., Bohlin, L., Larsson, D.G. J., Olsen, B., Chryssanthou, E. (2012). Antimicrobial activity of 
filamentous fungi isolated from highly antibiotic-contaminated river sediment. Infection Ecology and Epidemiology, 2, I-6. DOI: 10.3402/iee.v2i0.11591

9. Rančić , A., Soković, M., Karioti, A., Vukojević, J., Skaltsa, H. (2006). Isolation and structural elucidation of two secondary metabolites from the filamentous fungus Penicillium ochrochloron with antimicrobial activity. Environ. Toxicol. and Pharmacol., 22 (1), 80-84. DOI:10.1016/j.etap.2005.12.003

10. Petita, K.E., Mondeguerb, F., Roquebcrtc, M.F., Biarda, J.F., Pouchusa, Y.F. (2004). Detection of griseofulvin in a marine strain of Penicillium waksmanii by ion trap mass spectrometry. J Microbiol. Methods, 58, 59-65.

11. Ciharaci-Fathahm, E., Tajick-Ghanbary, M.A., Shahrokhi, N. (2014). Antimicrobial properties of Penicillium species isolated from agricultural soils of Northern Iran. Research Journal of Toxins, 6 (1), 1-7.

12. Daengrot, C., Rukachaisinkul, V., Tadpetch, K., Phongpaichi,t S., Bowornwiriyapan, K., Sakayarojc, J., Shend, X. (2016). Penicillanthone and penicillidic acids A - C from the soil-derived fungus Penicillium aculeatum PSU -RSPG105. RSC Advances, 46, 39530-40625.

13. Savchuk, Ya.I., Zaichenko, O.M., Tsiganenko, K.S. (2013). Antibiotic activity of some fungi. Mikrobiol. Z., 75 (5), 52-61.

14. Rubezhniak, I.G. Antibiotic and phytotoxic activities of cultural filtrates of some micromycete strains. (2018). Mikrobiol. Z., 80 (5), 90 -97. DOI: https://doi.org/10.15407/ microbiolj80.05.090

15. Bozoudi, D., Tsaltas, D. (2018). The multiple and versatile roles of Aureobasidium pullulans in the vitivinicultural sector. Fermentation, 4 (85), 1-15. DOI:10.3390/fermentation 4040085

16. Zain, M.E., Awaad, A.S., Razak, A.A., Maitland, D.J., Khaims, N.E., Sakhawy, M.A. (2009). Secondary metabolites of Aureobasidium pullulans isolated front Egyptian soil and their biological activity. J. Appl. Sci. Res., 5, 1582-1591.
17. McCormack, P.J., Wildman, H.G., Jeffries, P. (1994). Production of antibacterial compounds by phylloplane-inhabiting yeasts and yeast like fungi. Appl Environ. Microbiol., 60, 927-931.

18. Ferreira-Pinto, M.M., Moura-Guedes, M.C., Barreiro, M.G., Pais, I., Santos, M.R., Silva, M.J. (2006). Aureobasidium pullulans a biocontrol agent of blue mold in "Rocha" pear". Communications in agricultural and applied biological sciences, 71(3 Pt B), 973-978.

19. Zhang, D., Spadaro, D., Valente, S., Garibaldi, A., Gullino, M.L. (2012). Cloning, characterization, expression and antifungal activity of an alkaline serine protease of Aureobasidium pullulans PL5 involved in the biological control of postharvest pathogens. Int. J. of Food Microbiology, 153 (3), 453-464. DOI: 10.1016/j.ijfoodmicro.2011.12.016

20. Australian pesticides and veterinary medicines authority. (2017). Public release summary on the evaluation of the new active Aureobasidium pullulans (strains DSM 14940 and DSM 14941) in the product Botector Fungicide. Retrieved from URL: https://apvma.gov.au/sites/default/files/ publication/27401-82495_105881_-_botector_fungicide_-_prs_0.docx

21. Oliveira, S.M., Sena, K.X., Gusmio, N.B. (2009). Secondary metabolites produced by endophytic fungus Paecilomyces variotii Bainier with antimicrobial activity against Enterococcus faecalis. In: Proceedings of the II Intonational Conference on Environmental, Industrial and Applied Microbiology (BioMicroWorld2007), 2007 Nov 28 - Dec 1; University of Seville, Spain. Formatex Research Center, Spain, 519-22.

22. Wang, H., Hong, J., Yin, J., Moon, H.R., Liu, Y., Wei, X., Oh, D.C., Jung, J.H. (2015). Dimeric octaketide spiroketals from the jellyfish-derived fungus Paecilomyces variotii J08NF-1. J. Nat. Prod., 78 (11), 2832-2836.

23. Soman, A.G., Gloer, J.B., Wicklow, D.T. (1999). Antifungal and Antibacterial Metabolites from a Sclerotium-Colonizing Isolate of Mortierella vinacea. J. Nat. Prod., 62 (2), 386-388. 


\section{I. Г. РУбеЖНЯК (2020). АНТИБАКТЕРІАЛЬНА АКТИВНІСТЬ КУЛЬТУРАЛЬНИХ ФІЛЬTРАTІВ ШТАМІВ МІКРОМІЦЕTIB. BIOLOGICAL SYSTEMS: THEORY AND}

INNOVATION, 11(1): 42-49. http://journals.nubip.edu.ua/.

https://doi.org/10.31548/biologiya2020.01.042.

Анотація. Метою роботи був скринінг серед 125 итамів мікроміцетів, виділених із різних екологічних ніш, на антибактеріальну активність. Активність вивчалася з використанням різних тест-організмів - грамнегативних та грампозитивних бактерій. Показано, що тільки 64 досліджуваних итамів проявили антибактеріальну активність. Широким спектром антибіотичної дії характеризувалися досліджувані итами роду Аsp ergillus ma Penicillium. Штами роду Aureobasidium ma Paecilomусеs, такі як A. pullulans 41, P. variotii 68, також проявили антибактеріальний ефект на досліджувані бактерії. Встановлено, що итами М. vinacea проявляють антибактеріальний ефект на Staphylococcus aureus 904 ma E. coli 906. Більшість штамів не показали антибактеріальної дії. Найбільш перспективними для подальших досліджень є итами A. pullulans, P. variotii ma M. vinacea, які проявляють високу антибактеріальну активність та мало досліджені.

Ключові слова: мікроміцети, антибактеріальна активність, грамнегативні та грампозитивні бактерії. 